\title{
The star clusters of the Magellanic System
}

\author{
Basílio X. Santiago ${ }^{1}$ \\ ${ }^{1}$ Instituto de Física, Universidade Federal do Rio Grande do Sul \\ Caixa Postal 15051, Porto Alegre, RS, Brazil \\ email: santiago@if.ufrgs.br
}

\begin{abstract}
More than 50 years have elapsed since the first studies of star clusters in the Magellanic Clouds. The wealth of data accumulated since then has not only revealed a large cluster system, but also a diversified one, filling loci in the age, mass and chemical abundance parameter space which are complementary to Galactic clusters. Catalogs and photometric samples currently available cover most of the cluster mass range. The expectations of relatively long cluster disruption timescales in the Clouds have been confirmed, allowing reliable assessments of the cluster initial mass function and of the cluster formation rate in the Clouds. Due to their proximity to the Galaxy, Magellanic clusters are also well resolved into stars. Analysis of colour-magnitude diagrams (CMDs) of clusters with different ages, masses and metallicities are useful tools to test dynamical effects such as mass loss due to stellar evolution, two-body relaxation, stellar evaporation, cluster interactions and tidal effects. The existence of massive and young Magellanic clusters has provided insight into the physics of cluster formation. The magnitudes and colours of different stellar types are confronted with stellar evolutionary tracks, thus constraining processes such as convective overshooting, stellar mass-loss, rotation and pre main-sequence evolution. Finally, the Magellanic cluster system may contribute with nearby and well studied counterparts of recently proposed types of extragalactic clusters, such as Faint Fuzzies and Diffuse Star Clusters.
\end{abstract}

Keywords. Magellanic Clouds, galaxies: star clusters, galaxies: stellar content

\section{The census of star clusters in the Magellanic Clouds}

The first papers on star clusters in the Magellanic Clouds (MCs) date from over 50 years ago. They were concerned with detecting these objects and measuring their basic properties such as positions, apparent sizes and position angles. Figure 1 shows the evolution in the census of star clusters in the Clouds from these early papers to the present. The numbers shown were taken from references in the literature spanning the entire period (Kron 1956; Lindsay 1958; Shapley \& Lindsay 1963; Lyngå \& Westerlund 1963; Hodge \& Sexton 1966; Hodge 1986, 1988; Bica et al. 1999; Bica \& Dutra 2000; Bica et al. 2008). A steady increase in the number of known clusters can be seen, especially in the case of the Large Magellanic Cloud (LMC). For the Small Magellanic Cloud (SMC), the rise has been slower. This may, at least in part, reflect the fact that some of the earlier catalogs did not separate star clusters from stellar associations.

The latest catalog has just recently been published by Bica et al. (2008). It includes star clusters, associations and other extended objects. It contains a total of 9305 entries, 3740 of which have been classified as star clusters. The on-sky distribution of star clusters in Bica et al. (2008) is shown in Figure 2. We see that the main structural components of the Magellanic System are traced by these objects, including the LMC bar and outer ring, the SMC wing and the bridge.

One important issue is whether the current census of stars clusters is complete in the MCs. A bias against faint and/or compact star clusters should exist to some extent, as 


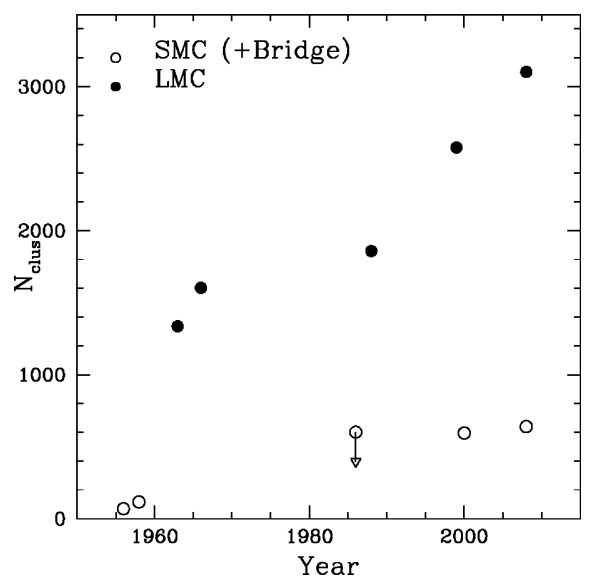

Figure 1. Census of known star clusters as a function of time. LMC: solid circles; SMC and Bridge: open circles.

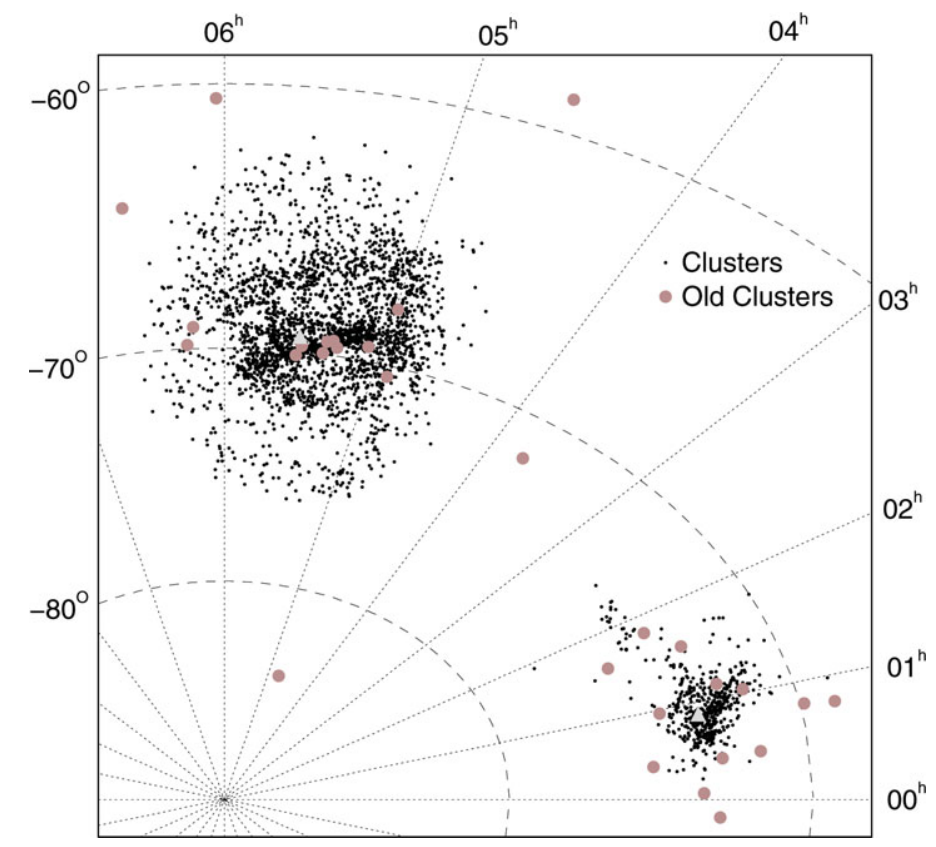

Figure 2. On-sky distribution of the 3740 star clusters in the Bica et al. (2008) catalogue, on a grid of equatorial coordinates. The larger dots are the clusters with estimated ages $\tau>4$ Gyr.

these objects are harder to detect or to separate from single bright stars. The apparent diameter function of star clusters from Bica et al. (2008) is shown in Figure 3, separately for the LMC, SMC and Bridge clusters. The clusters in the SMC and Bridge have been scaled to the LMC distance. The diameter function shows a power law behaviour at large diameters, reaching a peak at $D_{\text {app }} \simeq 0.6^{\prime}$ and then dropping sharply at the small diameter end. The distribution of tidal radii of Galactic globular clusters is also shown in the figure and has a similar shape. As these latter should make up an essentially complete sample, the authors conclude that this down turn is a real feature, rather than the result of a selection bias. 

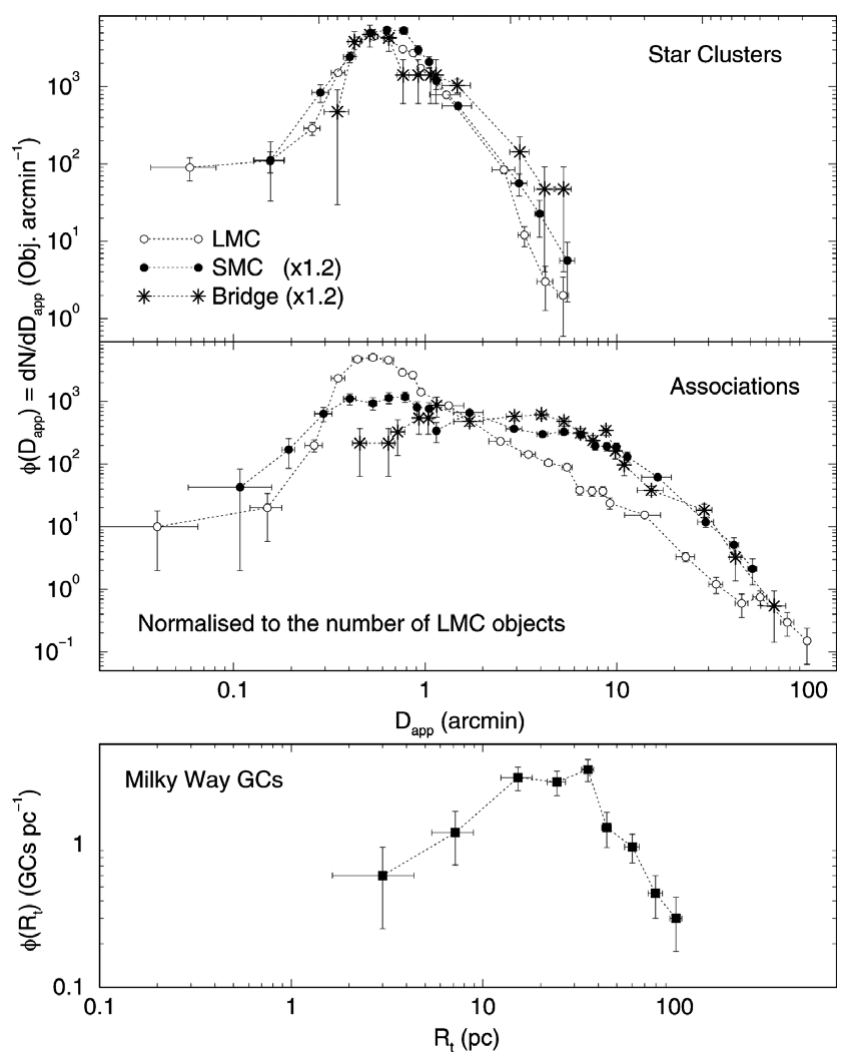

Figure 3. Top panel: apparent diameter function of star clusters of Bica et al. (2008). The LMC, SMC and Bridge clusters are shown in separate, as indicated. Middle panel: The diameter function of the 3326 stellar associations, again separately showing the LMC, SMC and Bridge systems. Lower panel: The distribution of tidal radii of Galactic globular clusters.

\section{Basic properties of the MC star cluster system}

Since the LMC and SMC are at distances of $\simeq 50 \mathrm{kpc}$ and $60 \mathrm{kpc}$, respectively, their system of star clusters can be studied in much more detail than those of more distant hosts. For instance, the photometric sample of LMC clusters by Hunter et al. (2003) is complete down to $M_{V} \simeq-3.5$. This is 4 magnitudes fainter than the absolute magnitude at the peak of the globular cluster luminosity function (GCLF) observed in other galaxies, especially in luminous early-type ones. Therefore, the LMC and SMC cluster systems are probed towards much lower masses than elsewhere.

Basic properties of hundreds of individual star clusters in both Clouds, such as ages, metallicities and masses, have been obtained from integrated photometry, integrated spectroscopy or colour-magnitude diagrams (CMDs) resulting from high-resolution imaging.

The age - metallicity relation (AMR) of rich LMC clusters compiled by Kerber et al. (2007) is shown in Figure 4. It allows us to discuss the main properties of the LMC cluster system. Rich clusters span a wide range in ages, $7 \leqslant \log \tau(\mathrm{yr}) \leqslant 10$. Most clusters younger than $\simeq 3$ Gyr have metallicities between half and one third of the solar value. Only about 15 rich clusters are older than $10 \mathrm{Gyr}$ and have $[\mathrm{Fe} / \mathrm{H}] \simeq-1.5$ or less, thus having properties similar to those of globular clusters (GCs) found in the Galactic halo. A noticeable feature is the so-called age gap, as only one cluster, ESO $121 \mathrm{SC03}$, is found 


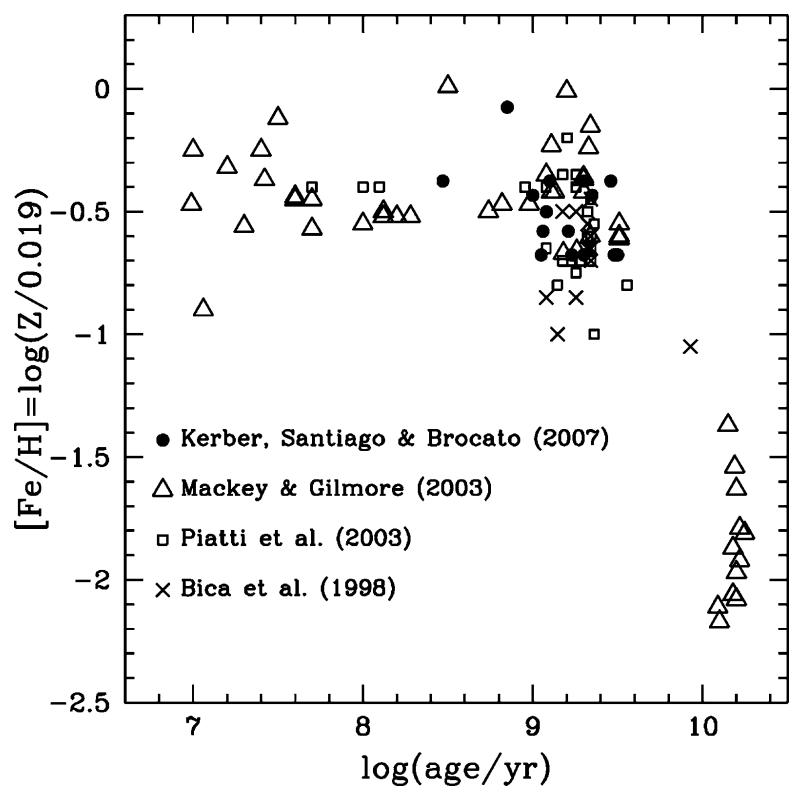

Figure 4. The age - metallicity relation for LMC clusters, taken from Kerber et al. (2007). The different symbols show clusters from different samples, as indicated.

in the range $3 \mathrm{Gyr} \leqslant \tau \leqslant 10 \mathrm{Gyr}$. This cluster has recently been studied by Xin et al. (2008), using Hubble Space Telescope Advanced Camera for Surveys (HST/ACS), and its age has been confirmed to fall in this interval.

The SMC cluster system is also rich and diversified in terms of ages and abundances. A recent AMR for the SMC can be found in Piatti et al. (2007). Despite the often large error bars in determined cluster ages, the SMC seems to have several examples of intermediate-age clusters, with no clear sign of an age gap. Another difference relative to the LMC is the absence of a significant population of rich, old and metal poor clusters similar to the Galactic GCs. Only NGC 121 has GC properties (Glatt et al. 2008).

\section{Age and mass distributions: constraining the cluster formation rate and initial mass function}

Large photometric surveys of both clouds are currently available, yielding magnitudes and colour for millions of stars and hundreds of star clusters (Massey 2002; Zaritsky et al. 1997; Hunter et al. 2003; Rafelski \& Zaritsky 2005).

The integrated magnitudes and colours of star clusters can be compared to those of single stellar populations, as predicted by different models (Leitherer et al. 1999; Kurth et al. 1999). As a result, age and mass estimates can be inferred for each cluster. The distribution of clusters with these quantities bears a lot of information on the cluster initial mass function (ICMF), the cluster formation history (CFR) and the timescale for cluster disruption.

As an example, Boutloukos \& Lamers (2003) have modelled the age distribution of star clusters. The model assumes that the ICMF is a power-law with cluster mass, $\propto m^{-2}$, and that the CFR has been uniform. Assuming also that the cluster disruption timescale, $t_{\mathrm{d}}$, is a power-law as a function of cluster mass, the model predicts that the number of star clusters per unit age should be described by a double power-law as a function of age, with a shallow slope at small ages and a steeper one for the older clusters. The slower 
drop at small ages results from fading: clusters become less luminous as they age, so that their number decreases in any magnitude limited sample. At larger ages, dynamical evolution leads to the disruption of less massive clusters, leading to a faster drop in the observed age distribution. This behaviour was in fact observed by Boutloukos \& Lamers (2003) in several galaxies with sizable cluster samples with age estimates, including the SMC. From the age distribution of the SMC clusters, they infer a disruption timescale

$$
t_{\mathrm{d}} \simeq 8\left(\frac{M}{10^{4} \mathrm{M}_{\odot}}\right)^{0.62} \mathrm{Gyr}
$$

Hunter et al. (2003) have obtained integrated magnitudes and colours for 939 SMC and LMC clusters using the images from the Massey (2002) survey. They have modelled the age and mass distributions and found evidence for cluster fading as well as an increase in maximum cluster mass for larger ages resulting from statistical sampling, from which they were able to constrain the ICMF slope.

De Grijs \& Anders (2006) re-analyzed the Hunter et al. (2003) data using a tool to compare clusters colours and ages to SSPs and applied the Boutloukos \& Lamers (2003) model in order to infer a $t_{\mathrm{d}}=8 \mathrm{Gyr}$ for $10^{4} \mathrm{M}_{\odot}$ LMC clusters, in agreement with the results for the SMC.

In brief, these studies quantitatively confirm that star clusters tend to live much longer in the Clouds than in the Galaxy as a result of slower disruption processes. We point out that, qualitatively, the differences in age distribution between the Galaxy and the Clouds is long known (Hodge 1988). These studies based on integrated photometry also constrained the ICMF slope and confirmed a roughly constant CFR in the MCs, apart from the age gap in the LMC.

\section{Magellanic star clusters in high resolution: structure and dynamics}

The HST has allowed individual star clusters to be resolved into stars and studied in detail (Brocato et al. 2001; Santiago et al. 2001). Detailed and self-consistent modelling of the colour-magnitude diagrams provides a strong tool for determining the physical properties of rich star clusters. Self-consistency should be understood in this context as the capacity to extract the relevant parameters, such as age, metallicity, distance, extinction and mass functions at different positions, all from the same data-set, without pre-fixing any of them. This may often be the most reliable approach considering the spatial depth of the two Clouds, the granularity of the dust distribution and the range in metallicities in these galaxies. Kerber et al. (2002) and Kerber \& Santiago (2005) presented a statistical method, based on a code from D. Valls-Gabaud, to model observed CMDs by comparing them to synthetic ones. The authors applied this tool to several LMC star clusters observed with HST's Wide Field and Planetary Camera 2 (HST/WFPC2). Similar techniques are also successfully applied to CMDs of field stars in the Clouds and other dwarf satellites of the Galaxy, in order to reconstruct the star formation history (SFH) in them (Hernandez et al. 2000; Javiel et al. 2005; Noël et. 2007).

The MCs are an excellent laboratory to observe and constrain dynamical effects on stellar systems, in particular star clusters. Dynamical and structural evolution can be observed and modelled from analysis of high resolution images in LMC and SMC clusters.

First, clusters respond to the evolution of their stars. Strong winds from massive stars and supernovae (SN) explosions lead to mass loss and expansion in young clusters, as observed by Bastian \& Goodwin (2006). Any gas not converted into stars before the first SN bursts will be swept out from the cluster. If the efficiency of star formation is low, mass loss can be severe, the remaining cluster becoming unbound and dispersing into the 

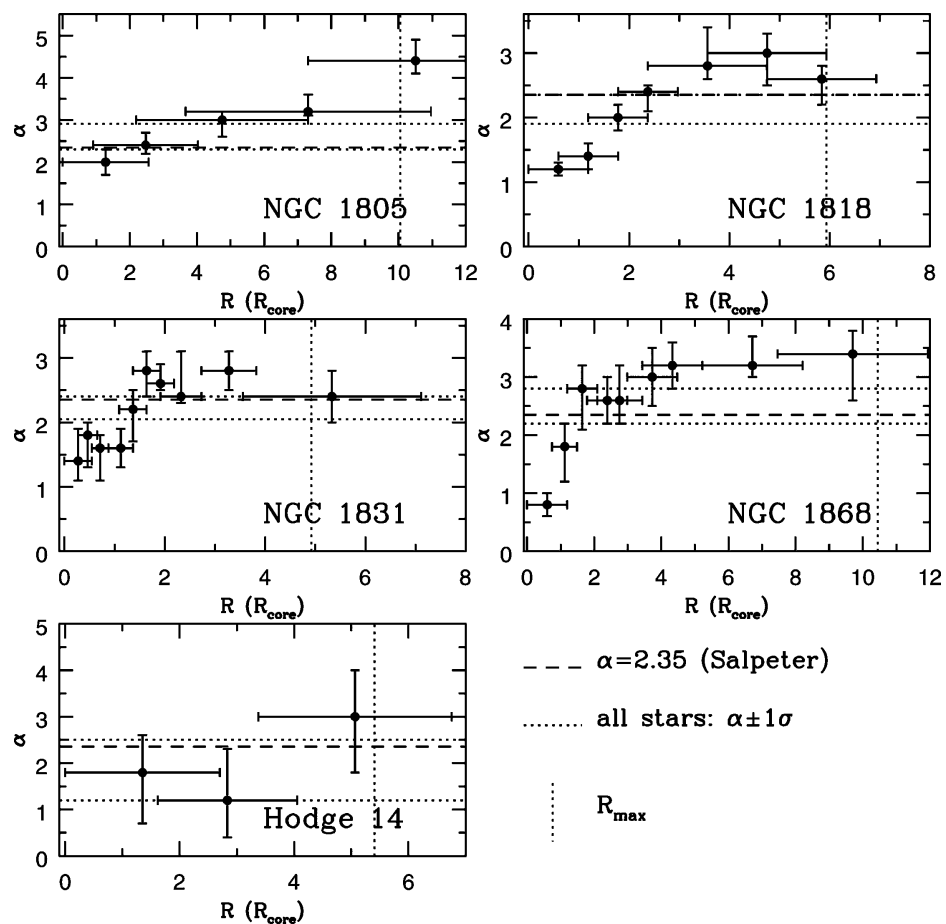

- - $\alpha=2.35$ (Salpeter)

all stars: $\alpha \pm 1 \sigma$

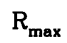

Figure 5. Variation in PDMF slope as a function of distance from the cluster centre for a sample of rich LMC clusters imaged with HST/WFPC2 and studied by Kerber \& Santiago (2006) and de Grijs et al. (2002). Each panel shows the slope $\alpha$ vs. $R$ relation for a different cluster, as indicated. The distances are in units of the core radius. The dashed line corresponds to $\alpha=2.35$ and the horizontal dotted lines indicate the $1 \sigma$ range around the global PDMF slope.

field at its early stages of evolution. This process is usually referred to as infant mortality. In fact, the infant mortality rate of Magellanic clusters, especially in the SMC, has been subject to a lot of recent controversy. Based on the decline in the number of clusters as a function of age, Chandar et al. (2006) have suggested that up to $90 \%$ of the young star clusters formed in the SMC do not survive the early stages. Kruijssen \& Lamers (2008) also needed a similarly large infant mortality rate to accommodate the observed age distributions of SMC clusters and field stars under the same general SFH. On the other hand, Gieles et al. (2007) and de Grijs \& Goodwin (2008) interpret the declining number of SMC clusters at young ages as being dominated by the effect of cluster fading in brightness in a magnitude-limited sample. More on infant mortality is discussed by R. de Grijs in these proceedings.

Second, two-body interactions among stars occur inside clusters, as the relaxation timescale is shorter than a Hubble time and may in fact be of a few Myr in compact and poor clusters. As a result of these interactions, more massive stars will tend to donate part of their orbital energy to lower mass ones; the former will tend to sink towards the gravitational potential well, whereas the latter will achieve less bound orbits, causing mass segregation inside the cluster. A fraction of the low mass stars will acquire escape velocity and leave the cluster (stellar evaporation).

Evidence for mass segregation and stellar evaporation is often observed in rich star clusters for which deep and high-resolution photometry is available, including young ones. Figure 5 shows that the present day mass function (PDMF) slopes of several LMC 


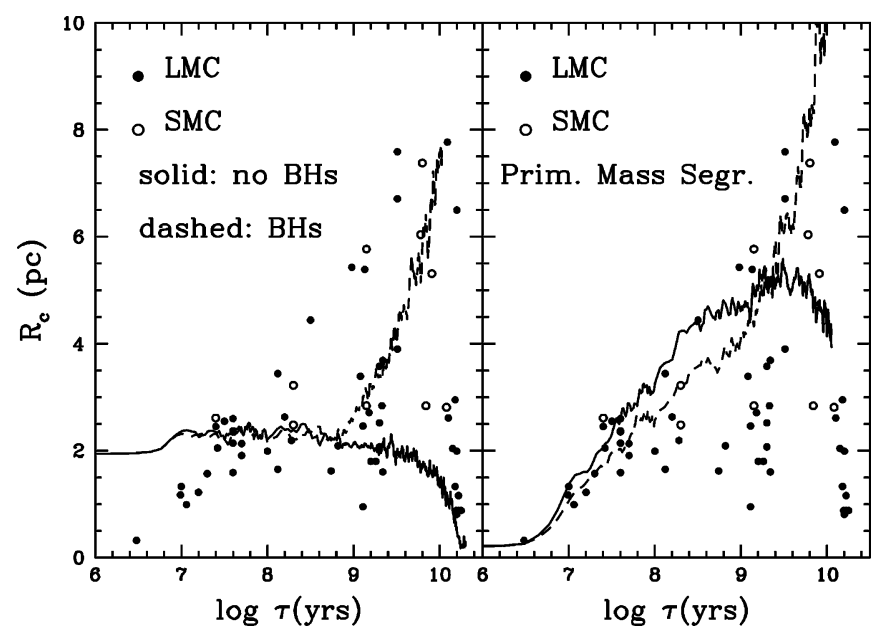

Figure 6. Left panel: Core radius $\left(R_{\mathrm{c}}\right.$, in parsecs) as a function of age $(\log \tau(\mathrm{yr}))$ for LMC and SMC star clusters. The data are from Mackey et al. (2003a,b). The lines show the expected evolution based on N-body simulations. Solid line: no heating from stellar degenerates; dashed line: heating included. Right panel: the data are the same as in the previous panel. The simulation results now include primordial mass segregation, again with and without heating by stellar black holes.

clusters modelled by Kerber \& Santiago (2006) increase steadily as a function of distance from the cluster centre. The slopes $\alpha$ result from power-law fits to the differential number of stars as a function of initial stellar mass, $d N / d m \propto m^{-\alpha}$, in different radial annuli around the cluster centre. At the largest distance bins, the trend flattens out or is even reversed in some cases, due to the loss of evaporating low mass stars from the cluster. Notice also that the global PDMF slopes are consistent with the Salpeter value $(\alpha=2.35)$.

For young enough clusters, the PDMF should reflect more closely the initial mass function (IMF). In particular, as the MCs are metal poorer than the Galaxy, and have a large system of young and rich clusters, mass function analysis in these clusters may help constrain variations in the IMF with environment. Kumar et al. (2008) have analyzed the mass function of 9 young star clusters in the LMC and found power-law slopes (in the mass range $2<m / \mathrm{M}_{\odot}<12$ ) consistent with one another for all but one of them. The slopes are also close to the Salpeter value, supporting the idea of a universal IMF. Schmalzi et al. (2008) have also found a nearly Salpeter value for the IMF slope in the SMC star forming regions NGC 602, in the mass range $1 \leqslant m / \mathrm{M}_{\odot} \leqslant 45$. On the other hand, at lower masses $\left(m / \mathrm{M}_{\odot}<1\right)$, the existence of a universal IMF is still in debate (see contribution by D. Gouliermis in these proceedings).

A clear evidence of structural evolution of star clusters is given by the relation between core size $\left(R_{\mathrm{c}}\right)$ and age. The core radius is determined by fitting an equilibrium model, such as the Elson et al. (1987) model, to the cluster surface brightness profile. The relation between $R_{\mathrm{c}}$ and age, using the data on rich LMC and SMC clusters from Mackey et al. $(2003 \mathrm{a}, \mathrm{b})$, is shown in Figure 6 . The mean core radius clearly increases with age, as does the spread around the mean. This shows not only that clusters change their structure with time but also that they do not follow a single path of core evolution. In fact, a large fraction of the clusters older than 10 Gyr shown in the Figure have very small cores, possibly having undergone core collapse.

Several mechanisms have been proposed to account for core evolution. Elson et al. (1989) explored the possibility that variations in the IMF from one cluster to another 
could lead to different rates of mass loss and core expansion, therefore explaining the spread in the $R_{\mathrm{c}}$-age relation. The mean trend towards larger cores has been tentatively explained as a sampling effect (Hunter et al. 2003), or as the result of dynamical heating of the core by binary stars (Wilkinson et al. 2003) or by stellar black holes (Mackey et al. 2008).

Mackey et al. (2008) used realistic N-body simulations of star clusters, in which the number of particles in the simulation is comparable to the number of real stars. They were able to reproduce the mean trend in core size with age by including the effect of core heating by black holes that sink to the cluster centre. The core evolution in simulations with and without the effect is shown in the left panel of Figure 6 . The upper boundary of the observed relation in LMC and SMC clusters can be reproduced for clusters with primordial mass segregation (right panel), especially in the case involving heating by black holes.

\section{MC star clusters as probes of stellar evolutionary theory}

Compared to the Galaxy, the Magellanic Clouds are gas rich and metal poor, thus providing a different environment where stars form and evolve. As such, they are a complementary and very useful laboratory to test stellar evolutionary theories. Observed cluster CMDs are particularly useful as they can be approximated as single stellar populations and compared to model isochrones. We here quote some recent examples focussed on very different stellar evolutionary phases.

Mucciarelli et al. (2007a,b) analyzed HST/ACS CMDs of two rich intemediate-age star clusters in LMC, namely NGC 1978 and NGC 1783. They compared several model expectations, with convective regions that exceed the classical one by values in the range $0 \leqslant \Lambda \leqslant 25 \%$, to their high-resolution CMDs. As a result, they favour a mild or large amount of convective overshooting $(\Lambda=0.10-0.25)$ in the intermediate mass stars at the main sequence turn-off, sub-giant (SGB) and red-giant (RGB) phases of these clusters. They also detected the elusive, but predicted, bump along the RGB sequence. Kerber \& Santiago, in these proceedings, have also tested different stellar evolution models, with and without overshooting, using a sample of 15 intermediate age clusters in the LMC.

Marigo \& Girardi (2007) have developed synthetic models to describe the late evolutionary stage called thermally pulsating asymptotic giant branch (TP-AGB), where significant variability, mass loss and dredge-up events take place. Their models were calibrated using the observed distribution of both luminosities and lifetimes of carbon rich and oxygen rich ( $\mathrm{C}$ and $\mathrm{M}$, respectively) AGB stars in the two Magellanic Clouds.

Hennekemper et al. (2008), again using HST/ACS, found a large number of pre-main sequence stars (PMS) in the young massive star forming region N66/NGC 346. Comparison of the observed PMS distribution in their CMD with model predictions by Siess et al. (2000) indicate the existence of two recent episodes of star formation. PMS stars, with $\tau \simeq 4 \mathrm{Myr}$, have also been recently detected by Carlson et al. (2007) in the star forming region NGC 602, located in the SMC wing.

\section{Spectroscopy of MC clusters: a bit of kinematics and abundances}

Radial velocities and detailed abundance analysis of MC clusters require use of spectroscopy. Recent detailed abundance studies of MC clusters can be found in Trundle et al. (2007), Hunter et al. (2007) and Mucciarelli et al. (2008). The first two analyzed over 100 B stars in several star clusters in the Galaxy and in the MCs, and with a large age span. They derived atmospheric parameters and photospheric abundances of 
$\mathrm{C}, \mathrm{N}, \mathrm{O}, \mathrm{Mg}, \mathrm{Si}$ and $\mathrm{Fe}$ and investigated the effect of evolutionary processes such as rotation, mass loss and binarity on the abundance of nitrogen.

Mucciarelli et al. (2008) studied spectra of 27 red giant stars located in 4 rich and intermediate-age LMC clusters. Their analysis yielded abundance ratios for about 20 atomic species, including $\alpha$, iron group and neutron capture elements.

Kinematic studies of the MC cluster systems date from the 1980s. Freeman et al. (1983) analyzed a sample of $59 \mathrm{LMC}$ clusters with individual radial velocities accurate to 10 $20 \mathrm{~km} \mathrm{~s}^{-1}$. They concluded that the LMC cluster system is consistent with a flattened disk rotating at $\simeq 40 \mathrm{kms}^{-1}$, although the disk geometry and systemic velocity was found to be different for young and old clusters. Schommer et al. (1992) used a larger cluster sample with more accurate velocities and concluded that all LMC clusters in their sample have a single disk kinematics. This result has been recently confirmed at $\simeq 2 \mathrm{~km} \mathrm{~s}^{-1}$ precision by Grocholski et al. (2006), who obtained metallicities and velocities from Ca II triplet lines for over 200 stars in 28 populous LMC clusters observed with the Very Large Telescope ( VLT). The authors also conclude that the LMC has no metallicity gradient.

For more on spectroscopic studies of star clusters in both Clouds, we refer to the contributions by A. Ahumada, G. Bosch, A. Grocholski and A. Mucciarelli to this symposium.

\section{Comparison with other star cluster systems}

Knowledge on extragalactic clusters has increased immensely in the last two decades. Among the most important breakthroughs are the universal (or nearly so) GCLF, the existence of bimodality in the distribution of globular cluster colours and, more recently, the ability to measure cluster sizes. Correlations involving cluster luminosities, colours, sizes, location and host properties provide insight into the process of galaxy and cluster formation and evolution.

An interesting and relatively unexplored piece of information is the size distribution of star clusters. The distributions of half-light radii $\left(R_{\text {eff }}\right)$ of massive clusters in the Magellanic Clouds and in the Galaxy are shown in the left panel of Figure 7. The figure

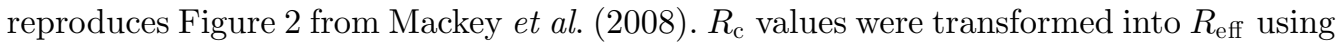
the relation quoted by Larsen (2001). For the Clouds, only clusters older than $\tau>7$ Gyr are shown, in order to make them more comparable to their Galactic counterparts, which are all globular clusters. For the Galaxy, clusters located closer than $R_{\mathrm{g}}=15 \mathrm{kpc}$ to the centre were eliminated from the figure, as these suffer stronger tidal effects, not occuring in the Clouds, and thus tend to be smaller $\left(R_{\mathrm{eff}} \simeq 3 \mathrm{pc}\right)$. The two distributions are very similar. The peak typical of Galactic globular clusters in the inner halo is still the dominant one. Two other peaks, at $R_{\text {eff }} \simeq 7$ pc and at $R_{\text {eff }} \simeq 15$ pc are also seen.

The right panel shows the distribution of star clusters with measured sizes in NGC 1380, which is a lenticular galaxy in the Fornax galaxy cluster. The data are from Chies-Santos et al. (2007). There is no cut in distance from the host centre, which likely explains the higher fraction of clusters at $R_{\text {eff }} \simeq 3$ pc. Interestingly, secondary peaks, similar to but at smaller radii than those in the Galaxy and in the Clouds, are also present.

The existence of clusters more extended than typical globulars may be evidence of distinct populations of star clusters, possibly with different formation and evolution histories. In well studied luminous early-type galaxies, which are the ones that harbour the richest cluster systems, extended clusters may also be distinguished from the more common globulars in terms of other properties. Larsen \& Brodie (2000) and Brodie \& Larsen (2002), for instance, found extended clusters with $7 \leqslant R_{\text {eff }} \leqslant 15$ pc in two nearby lenticular galaxies. These were named Faint Fuzzies, as they also tend to be underluminous 


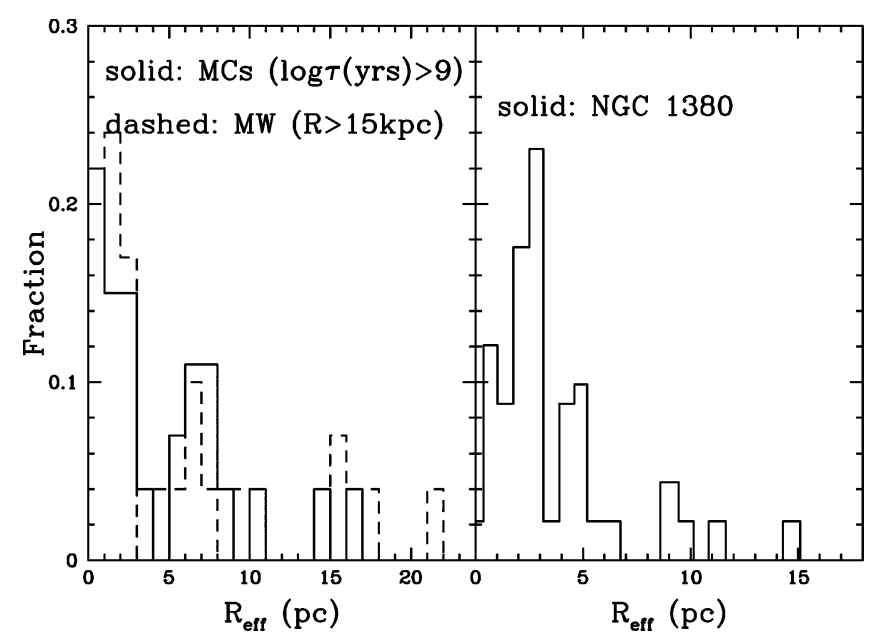

Figure 7. Left panel: Distribution of half-light radii of clusters in the Magellanic Clouds (solid) and in the Galaxy (dashed). Only clusters with $\log \tau(\mathrm{yr})>9$ in the Mackey et al. (2003a,b) samples are included. The Galactic sample comes from Harris. (1996) (and its 2003 web update) and includes only clusters farther than $15 \mathrm{kpc}$ from the Galactic centre, in order to avoid clusters more strongly affected by tidal effects. Right panel: distribution of $R_{\text {eff }}$ for clusters in NGC 1380, which is a luminous S0 galaxy in the Fornax cluster. The data are from Chies-Santos et al. (2007).

compared to the dominant cluster population. Their metallicities were estimated espectroscopically, yielding $[\mathrm{Fe} / \mathrm{H}] \sim-0.6$. Finally, Burkert et al. (2005) found evidence that Faint Fuzzies in NGC 1023 are kinematically connected in a ring-like structure.

Diffuse star clusters (DSCs) may constitute another recently found class. These were found by Peng et al. (2006) in the ACS Virgo Cluster survey (Côté et al. 2004). Several of luminous early-type galaxies, most of them S0s, have a large population of extended clusters of much lower surface brightness $\left(\mu_{\mathrm{g}}>20 \mathrm{mag} \operatorname{arcsec}^{-2}\right)$ than typical globulars. The DSCs are also redder than typical globular clusters, often having the same colour as the difuse light from the host.

A useful tool used by Peng et al. (2006) to separate different types of clusters is the luminosity-size diagram. The $M_{\mathrm{V}}-R_{\text {eff }}$ plane is shown in Figure 8. Massive clusters in 3 markedly different environments for cluster formation and evolution, the Galaxy, the Magellanic Clouds and NGC 1380, are shown in the Figure. The data on $M_{\mathrm{V}}$ are from the same references as the sizes. The dominant population in the Galaxy and in NGC 1380 is made up of clusters with $R_{\text {eff }}=1-4$ pc and $-9 \leqslant M_{\mathrm{V}} \leqslant-6$. Only a handful of such globular-like clusters are found in the Clouds. The Clouds also contribute little to the FFs box, shown in the lower right of each panel. In fact, most of the clusters in the LMC and SMC tend to follow the low-surface brightness line used by Peng et al. (2006) to characterize the DSCs. This means that the LMC and SMC have a large number of massive clusters that can be considered as structural counterparts to DSCs, although they do not necessarily share the other properties associated to these objects.

\section{Acknowledgements}

I thank the SOC for the invitation to give this review talk. C. Bonatto, L. Kerber and D. Mackey kindly provided figures and data included in this review. Useful discussions preceding my conference presentation were carried out with colleagues in my home institution, especially O. Kepler, M. Pastoriza, E. Bica and C. Bonatto. 


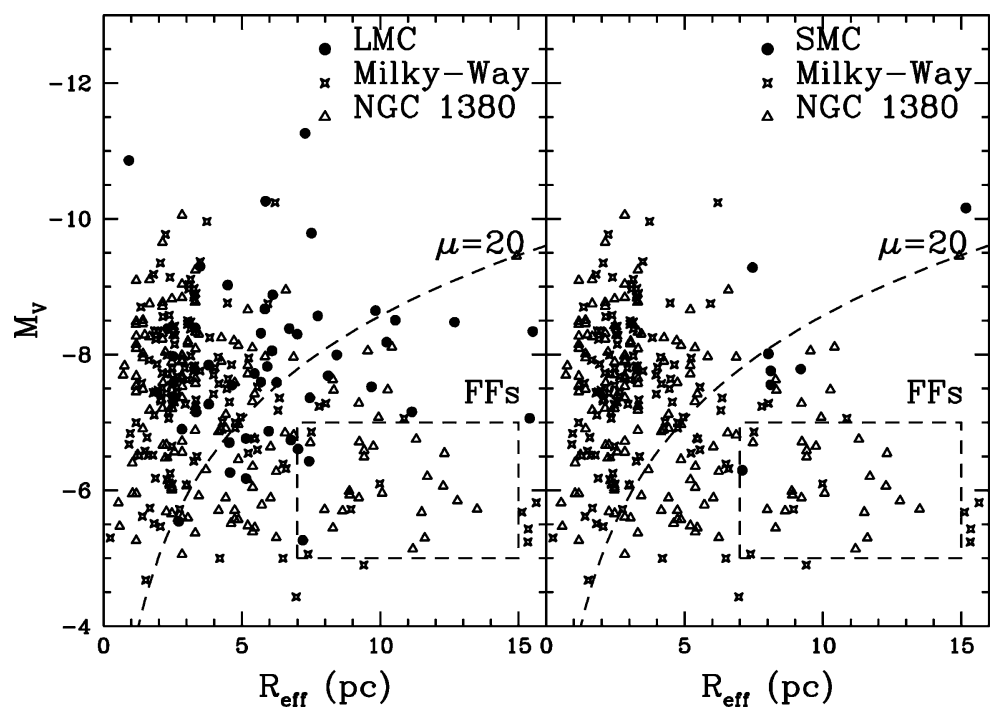

Figure 8. Left panel: $M_{\mathrm{V}}$ vs. $R_{\text {eff }}$ relation for star clusters in the LMC (solid circles), the Galaxy (stars) and in the luminous S0 in Fornax galaxy clusters, NGC 1380 (triangles). Right panel: the same as in the previous panel, but now the SMC clusters are compared to those in the Galaxy and in NGC 1380. In both panels, the dashed box in the lower right is the region occupied by faint fuzzies. The dashed line corresponds to a surface brightness of $\mu_{\mathrm{V}}=20$ mag $\operatorname{arcsec}^{-2}$, where DSCs preferentially lie.

\section{References}

Bastian, N. \& Goodwin, S. 2006, MNRAS, 369, L9

Bica, E., Geisler, D., \& Dottori, H. 1998, AJ, 116, 723

Bica, E., Schmitt, H., Dutra, C., \& Oliveira, H. 1999, AJ, 117, 238

Bica, E. \& Dutra, C. 2000, AJ, 119, 1214

Bica, E., Bonatto, C., Dutra, C., \& Santos, J. 2008, MNRAS, 389, 678

Boutloukos, S. \& Lamers, H. 2003, MNRAS, 338, 717

Brocato, E., Di Carlo, E., \& Menna, G. 2001, A\&A, 374, 523

Brodie, J. \& Larsen, S. 2002, AJ, 124, 1410

Burkert, A., Brodie, J., \& Larsen, S. 2005, ApJ, 628, 231

Carlson, L., Sabbi, E., Sirianni, M., et al. 2007, ApJ, 665, L109

Chandar, R., Fall, S. M., \& Whitmore, B. 2006, ApJ, 650, L111

Chies-Santos, A., Santiago, B., \& Pastoriza, M. 2007, A\& A, 467, 1003

Côté, P., Blakeslee, J., Ferrarese, L., et al. 2004, ApJS, 153, 223

de Grijs, R., Gilmore, G., Mackey, A., Wilkinson, M., Beaulieu, S., Johnson, R., \& Santiago, B. 2002, MNRAS, 337, 597

de Grijs, R. \& Anders, P. 2006, MNRAS, 366, 295

de Grijs, R. \& Goodwin, S. 2008, MNRAS, 383, 1000

Elson, R., Fall, M., \& Freeman, K. 1987, ApJ, 323, 54

Elson, R., Freeman, K., \& Lauer, T. 1989, ApJ, 347, L69

Freeman, K., Illingworth, G., \& Oemler, A. 1983, ApJ, 272, 488

Gieles, M., Lamers, H., \& Portegies Zwart, S. 2007, ApJ, 668, 268

Glatt, K., Gallagher, J., Grebel, E., et al. 2008, AJ, 135, 1106

Grocholski, A., Cole, A., Sarajedini, A., Geisler, D., \& Smith, V. 2006, AJ, 132, 1630

Harris, W. 1996, AJ, 112, 1487

Hennekemper, E., Gouliermis, D., Henning, T., Brandner, W., \& Dolphin, A. 2008, ApJ, 672, 914 
Hernandez, X., Valls-Gabaud, D., \& Gilmore, G. 2000, MNRAS, 317, 831

Hodge, P. \& Sexton, J. 1966, AJ, 71, 363

Hodge, P. 1986, PASP, 98, 1113

Hodge, P. 1988, PASP, 100, 576

Hunter, D., Elmegreen, B., Dupuy, T., \& Mortonson, M. 2003, AJ, 126, 1836

Hunter, I., Dufton, P., Smartt, S., et al. 2007, A\&A, 466, 277

Javiel, S., Santiago, B., \& Kerber, L. 2005 A $\mathscr{E} A$, 431, 73

Kerber, L., Santiago, B., Castro, R., \& Valls-Gabaud, D. 2002, A\& A, 390, 121

Kerber, L. \& Santiago, B. 2005, A\&SA, 435, 77

Kerber, L. \& Santiago, B. 2006, A\&A A, 452, 155

Kerber, L., Santiago, B., \& Brocato, E. 2007, A\&A, 462, 139

Kron, G. 1956, PASP, 68, 125

Kruijssen, J. \& Lamers, H. 2008, in J. G. Funes \& E. M. Corsini (eds.), Formation and Evolution of Galaxy Disks, ASP-CS, 396, p. 149

Kumar, B., Sagar, R., \& Melnick, J. 2008, MNRAS, 386, 1380

Kurth, O., Fritze-v, A., \& Fricke, K. 1999, A $\mathscr{E} A S, 139,19$

Larsen, S. \& Brodie, J. 2000, AJ, 120, 2938

Larsen, S. 2001, $A J, 122,1782$

Leitherer, C., Schaerer, D., Goldader, J., et al. 1999, ApJS, 123, 3

Lindsay, E. 1958, MNRAS, 118, 172

Lyngå, G. \& Westerlund, B. 1963, MNRAS, 127, 31

Mackey, A. \& Gilmore, G. 2003a, MNRAS, 338, 85

Mackey, A. \& Gilmore, G. 2003b, MNRAS, 338, 120

Mackey, A., Wilkinson, M., Davies, M., \& Gilmore, G. 2008, MNRAS, 386, 65

Marigo, P. \& Girardi, L. 2007, A\&A, 469, 239

Massey, P. 2002, ApJS, 141, 81

Mucciarelli, A., Ferraro, F., Origlia, L., \& Fusi Pecci, F. 2007a, AJ, 133, 2053

Mucciarelli, A., Origlia, L., \& Ferraro, F. 2007b, AJ, 134, 1813

Mucciarelli, A., Carretta, E., Origlia, L., \& Ferraro, F. 2008, AJ, 136, 375

Noël, N., Aparício, A., Gallart, C., Hidalgo, S., Costa, E., Méndez, R. 2007, in A. Vazdekis, \& R.F. Peletier (eds.), Stellar Populations as Building Blocks of Galaxies, IAU Conf.Proc. 241 (Cambridge: CUP), p. 373

Peng, E., Coté, P., Jordán, A., et al. 2006, ApJ, 639, 838.

Piatti, A., Bica, E., Geisler, D., \& Clariá, J. 2003, MNRAS, 344, 965

Piatti, A., Sarajedini, A., Geisler, D., Gallart, C., \& Wischnjewsky, M. 2007, MNRAS, 382, 1203

Rafelski, M. \& Zaritsky, D. 2005, AJ, 129, 2701

Santiago, B., Beaulieu, S., Johnson, R., \& Gilmore, G. 2001, A\&A, 369, 74

Schmalzi, M., Gouliermis, D., Dolphin, A., \& Henning, T. 2008, ApJ, 681, 290

Schommer, R., Olszewski, E., Suntzeff, N., \& Harris, H. 1992, AJ, 103, 447

Siess, L., Dufour, E., \& Forestini, M. 2000, A\& $A$, 358, 593

Shapley, H. \& Lindsay, E. 1963, IrAJ, 6, 74

Trundle, C., Dufton, P., Hunter, I., Evans, C., Lennon, D., Smartt, S., \& Ryans, R. 2007, A\&A, 471,625

Wilkinson, M., Hurley, J., Mackey, A., Gilmore, G., \& Tout, C. 2003, MNRAS, 343, 1025

Xin, Y., Deng, L., de Grijs, R., Mackey, A., \& Han, Z. 2008, MNRAS, 384, 410

Zaritsky, D., Harris, J., \& Thompson, I. 1997, AJ, 114, 1002 